\title{
A Staining Method for Easy Detection of Eosinophilic Globules in Kaposi's Sarcoma
}

\author{
Masachika Senba and Hideyo Itakura \\ Department of Pathology, Institute of Tropical Medicine, \\ Nagasaki University, Nagasaki 852
}

Senba, M. and Itakura, H. A Staining Method for Easy Detection of Eosinophilic Globules in Kaposi's Sarcoma. Tohoku J. exp. Med., 1987, 152 (2), 209-210 — Eosinophilic globules provide an important clue for diagnosis of Kaposi's sarcoma (KS). These globules were stained with periodic acid Schiff (PAS), periodic acid Schiff reagent after diastase digestion, phosphotungstic acid hematoxylin (PTAH) and autofluorescence under ultraviolet illumination. By these methods, however, eosinophilic globules in KS could not be readily identified. Therefore, we developed a new procedure for rapid identification of eosinophilic globules in KS tissues. eosinophilic globules; Kaposi's sarcoma

Blumenfeld and co-workers (1985) stated that eosinophilic globules of varying sizes could be exclusively found in cases of Kaposi's sarcoma (KS). Histochemical characteristics of eosinophilic globules in KS were reported previously (Senba et al. 1984, 1986 ; Senba 1985). These globules were stained pink with hematoxylin-and-eosin, purple with periodic acid Schiff (PAS), purple with periodic acid Schiff reagent after diastase digestion, blue with phosphotungstic acid hematoxylin (PTAH), and emitted yellowish autofluorescence under ultraviolet illumination. However, these staining procedures could not allow to identify eosinophilic globules easily, because background tissue elements were also stained concurrently. Both eosinophilic globules and erythrocytes were also autofluorescent. We propose a new procedure for ready identification of eosinophilic globules in KS.

Cutaneous type of KS in Africa was used. The materials were fixed in formalin and embedded in paraffin. The steps involved in the eosinophilic globule staining technique are as follows: (1) Deparaffinize and hydrate to distilled water. (2) Treat with celestine blue solution for $5 \mathrm{~min}$. Celestine blue solution: Dissolve $5 \mathrm{~g}$ ferric ammonium sulfate in $100 \mathrm{ml}$ distilled water, then dissolve $0.5 \mathrm{~g}$ celestine blue in this solution and bring to a boil for 3 to $5 \mathrm{~min}$. Cool and add $15 \mathrm{ml}$ glycerin and $1 \mathrm{ml}$ hydrochloric acid. (3) Wash in running water. (4) Treat with saturated picric acid in $80 \%$ alcohol for 1 min. (5) Wash in running water. (6) Treat with acid fuchsin solution for 5 min. Acid fuchsin solution: Dissolve $1 \mathrm{~g}$ acid fuchsin (Merck, Art, 7629) in $100 \mathrm{ml}$ distilled water and add $1 \mathrm{ml}$ glacial acetic acid. (7) Wash in running water. (8) Treat with differentiating solution for 5 min. Differentiating solution: Dissolve $10 \mathrm{~g}$ phosphotungstic acid and $1 \mathrm{~g}$ picric acid in $100 \mathrm{ml}$ of $80 \%$ alcohol solution. (9) Wash in running water. (10) Treat with aniline blue solution for $5 \mathrm{~min}$. Aniline blue solution: In $100 \mathrm{ml}$ of distilled water, dissolve $0.5 \mathrm{~g}$ aniline blue

Received March 30, 1987; accepted for publication May 6, 1987.

Supported in part by Grant No. 62921044 from the Ministry of Education, Science and Culture of Japan. 


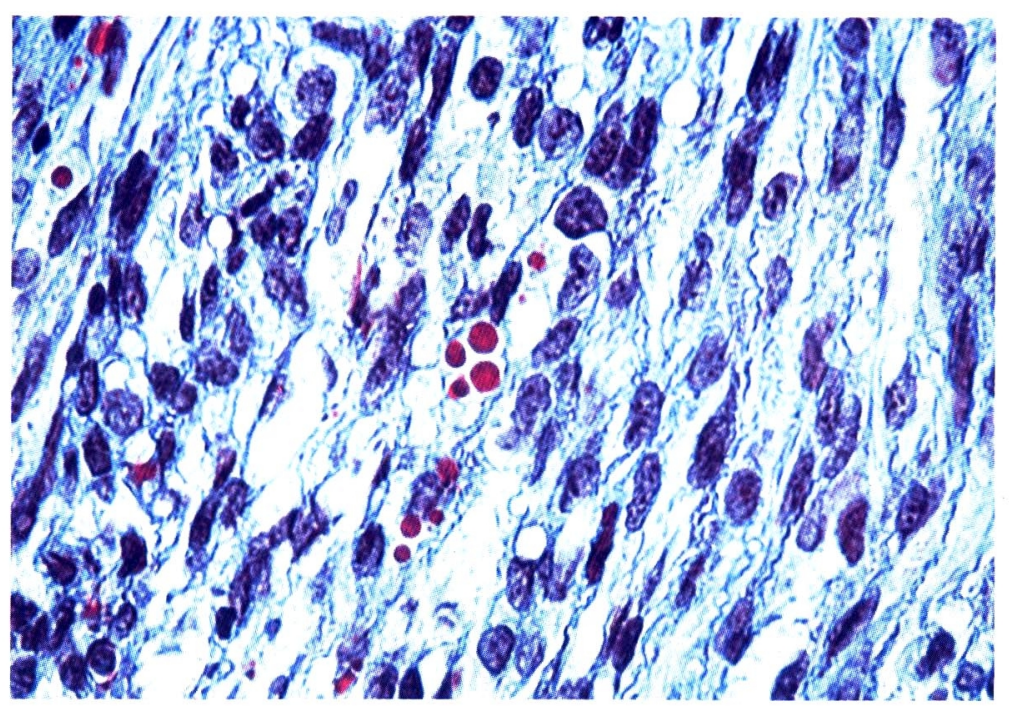

Fig. 1. Eosinophilic globules were seen in Kaposi's sarcoma by the acid fuchsin method. Original magnification $\times 320$.

(Merck, Art. 1279) and add $5 \mathrm{ml}$ glacial acetic acid. (11) Dehydrate, clear and mount.

Eosinophilic globules and fibrin were stained red (Fig. 1). Nuclei were stained blue. Collagen fibers were stained blue. Erythrocytes were stained yellow. By this procedure, these eosinophilic globules were more readily distinguishable from those stained with other methods.

The eosinophilic globules seen in KS were very similar at the light microscopic level to the globular bodies found in yolk sac tumors, alpha-1-antitrypsin deficiency, hepatocellular carcinoma, lung carcinoma, glioblastoma, and adrenal medulla. They have also similar histochemical properties to those of KS, such as periodic acid Schiff (PAS) and periodic acid Schiff reagent after diastase digestion, phosphotungstic acid hematoxylin (PTAH), and autofluorescence positive.

The mechanism of the eosinophilic globules and acid fuchsin dye staining reaction in the solution might be considered. The eosinophilic globules contain positively charged substances. On the other hand, electric charge of acid fuchsin is negative. It is, therefore, that the eosinophilic globules may react with acid fuchsin dye because of coupling of the electric charge.

\section{References}

1) Blumenfeld, W., Egbert, B.M. \& Sagebiel, R.W. (1985) Differential diagnosis of Kaposi's sarcoma. Arch. Path. Lab. Med., 109, 123-127.

2) Senba, M. (1985) Autofluorescence of eosinophilic globules in Kaposi's sarcoma. Arch. Path. Lab. Med., 109, 703.

3) Senba, M., Itakura, H., Toriyama, K. \& Uzuta, F. (1984) Periodic acid Schiff (PAS) and phosphotungstic acid hematoxylin (PTAH) positive materials in cutaneous type of Kaposi's sarcoma. Trop. Med., 26, 87-91.

4) Senba, M., Itakura, H., Yamashita, H. \& Huang, H.J. (1986) Eosinophilic globules in Kaposi's sarcoma: A histochemical, immunohistochemical, and ultrastructural study. Acta path. jap., 36, 1327-1333. 